\title{
Biomechanical Evaluation of Amorphous Calcium Phosphate Coated TNTZ Implants Prepared Using a Radiofrequency Magnetron Sputtering System
}

\author{
Naru Shiraishi ${ }^{1}$, Rong $\mathrm{Tu}^{2}$, Risa Uzuka ${ }^{3, *}$, Takahisa Anada ${ }^{4}$, Takayuki Narushima ${ }^{5}$, \\ Takashi Goto ${ }^{2}$, Mitsuo Niinomi ${ }^{2}$, Keiichi Sasaki ${ }^{3}$ and Osamu Suzuki ${ }^{4}$ \\ ${ }^{1}$ Research Unit for Interface Oral Health Science, Graduate School of Dentistry, Tohoku University, Sendai 980-8577, Japan \\ ${ }^{2}$ Institute for Materials Research, Tohoku University, Sendai 980-8575, Japan \\ ${ }^{3}$ Division of Advanced Prosthetic Dentistry, Graduate School of Dentistry, Tohoku University, Sendai 980-8577, Japan \\ ${ }^{4}$ Division of Craniofacial Function Engineering, Graduate School of Dentistry, Tohoku University, Sendai 980-8577, Japan \\ ${ }^{5}$ Department of Materials Processing, Graduate School of Engineering, Tohoku University, Sendai 980-8579, Japan
}

Ti-29Nb-13Ta-4.6Zr alloy (TNTZ), a new $\beta$-type Ti alloy, has excellent advantages as a biomaterial, such as low Young's modulus and cytotoxicity, and the absence of allergens. However, it is unclear whether TNTZ can achieve sufficient osseointegration for it to be used as a dental implant. The effectiveness of surface modification of TNTZ implants by radiofrequency (RF) magnetron sputtering is also unclear. We investigated the biomechanical behaviors of TNTZ implants in vivo, using cylindrical implants of four types: pure Ti, TNTZ, and pure Ti and TNTZ coated with amorphous calcium phosphate (ACP). The implants were inserted in rat femurs, and the femurs were subjected to biomechanical analyses after various time intervals. The results suggest that TNTZ implants can achieve osseointegration similar to that of pure $\mathrm{Ti}$, and that surface modification with ACP by RF magnetron sputtering improves osseointegration, especially in the later stages of healing. [doi:10.2320/matertrans.M2012078]

(Received March 6, 2012; Accepted May 8, 2012; Published June 20, 2012)

Keywords: amorphous calcium phosphate, dental implant, osseointegration, push-in test, Ti-29Nb-13Ta-4.6Zr (TNTZ)

\section{Introduction}

Ti-29Nb-13Ta-4.6Zr alloy (TNTZ) has excellent advantages as a biomaterial, such as a low Young's modulus (around $60 \mathrm{GPa}$ ) and the absence of allergenic elements. ${ }^{1)}$ Niinomi et al. reported that the cytotoxicity of TNTZ was nearly the same as that of commercially available pure $\mathrm{Ti}$ (cpTi) and greater than that of a conventional biomaterial, Ti-6Al-4V. ${ }^{2,3)}$ When TNTZ was inserted into rabbit lateral femoral condyles, newly formed bones were observed at the interface between the implant and the surrounding tissue. ${ }^{4)}$ In the field of orthopedics, a significant advantage of a low Young's modulus is inhibition of bone atrophy and enhanced bone remodeling, which prevents stress shielding. ${ }^{5-7)}$ These reports suggested to us that implants with a low Young's modulus would mechano-biologically adapt to the adjacent bone and prevent loss of osseointegration over time.

In dental implant treatment, osseointegration at the $\mathrm{Ti}-$ bone interface is essential for the success of the treatment; this is defined as direct integration of the implant with the bone. ${ }^{8)}$ From the biomechanical viewpoint, occlusal forces on the implant are directly transmitted to the adjacent bone as a mechanical stress. Some studies have suggested that occlusal overload may contribute to implant bone loss and/or loss of integration of successfully integrated implants. ${ }^{9-13)}$ It has been hypothesized that the use of TNTZ for dental implants could deal with these biomechanical issues by improving the stress distribution in the peri-implant bone.

The elements and proportions in TNTZ are different from those in cpTi, and it expected that the osseointegration ability of TNTZ is less than that of cpTi. We thought that surface modification of TNTZ by plasma spraying, sputtering,

*Graduate Student, Tohoku University or electrophoretic deposition would improve osseointegration. ${ }^{14)}$ Narushima et al. previously reported that amorphous calcium phosphate (ACP) coating using a radiofrequency (RF) magnetron sputtering system could be achieved at room temperature; the coating was a very thin layer (within $500 \mathrm{~nm}$ ) and had sufficient bonding strength (over $60 \mathrm{MPa}){ }^{15)}$ To assess the biocompatibility of the coating with bone, threaded implants were inserted in the mandibles of beagle-dogs and in rabbit femurs. The bone-implant contact of ACP-coated cpTi implants was significantly higher than those of non-coated implants. ${ }^{16)}$ Moreover, the removal torque values of ACP-coated Ti-6Al-4V implants were significantly higher than those of non-coated implants. ${ }^{17}$

The aim of this study is to confirm the osseointegration ability of TNTZ implants and to investigate the effectiveness of surface modification of TNTZ implants by RF magnetron sputtering.

\section{Materials and Methods}

All procedures were approved by the Animal Research Committee of Tohoku University.

\subsection{Implant design}

Non-screw-type, cylindrical implants $(\varphi 1.0 \times 2.0 \mathrm{~mm})$ made from cpTi and TNTZ were prepared. Each implant was polished with wet abrasive paper (P1500, Sankyo-Rikagaku Co, Saitama, Japan). After ultrasonic cleaning with $99.5 \%$ ethanol, the surface was coated with ACP using an RF magnetron sputtering system with $\beta$-TCP targets (MS-320, Universal Systems Co., Ltd., Tokyo, Japan). Four types of implants, namely cpTi, ACP-coated cpTi (ACP-cpTi), TNTZ and ACP-coated TNTZ (ACP-TNTZ), were prepared for this study. 


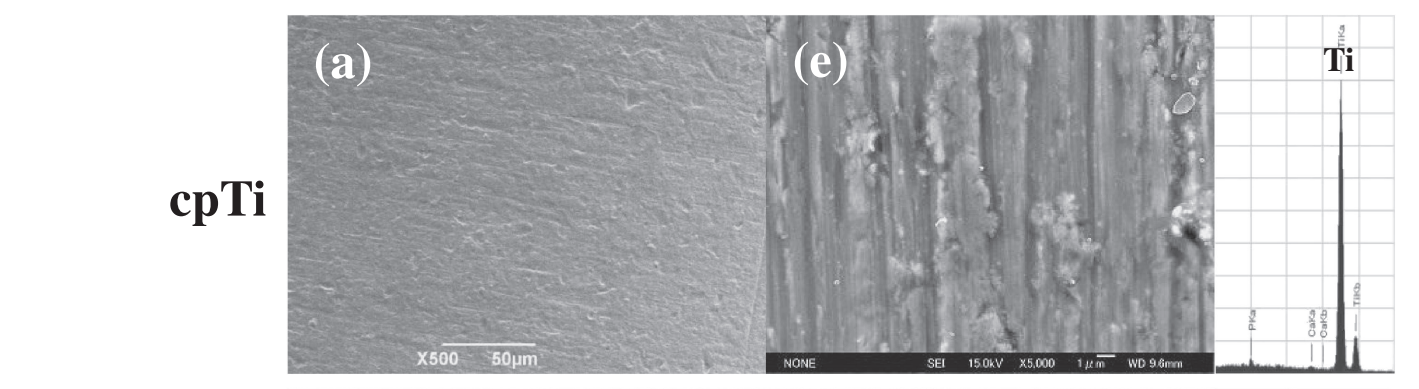

(i)
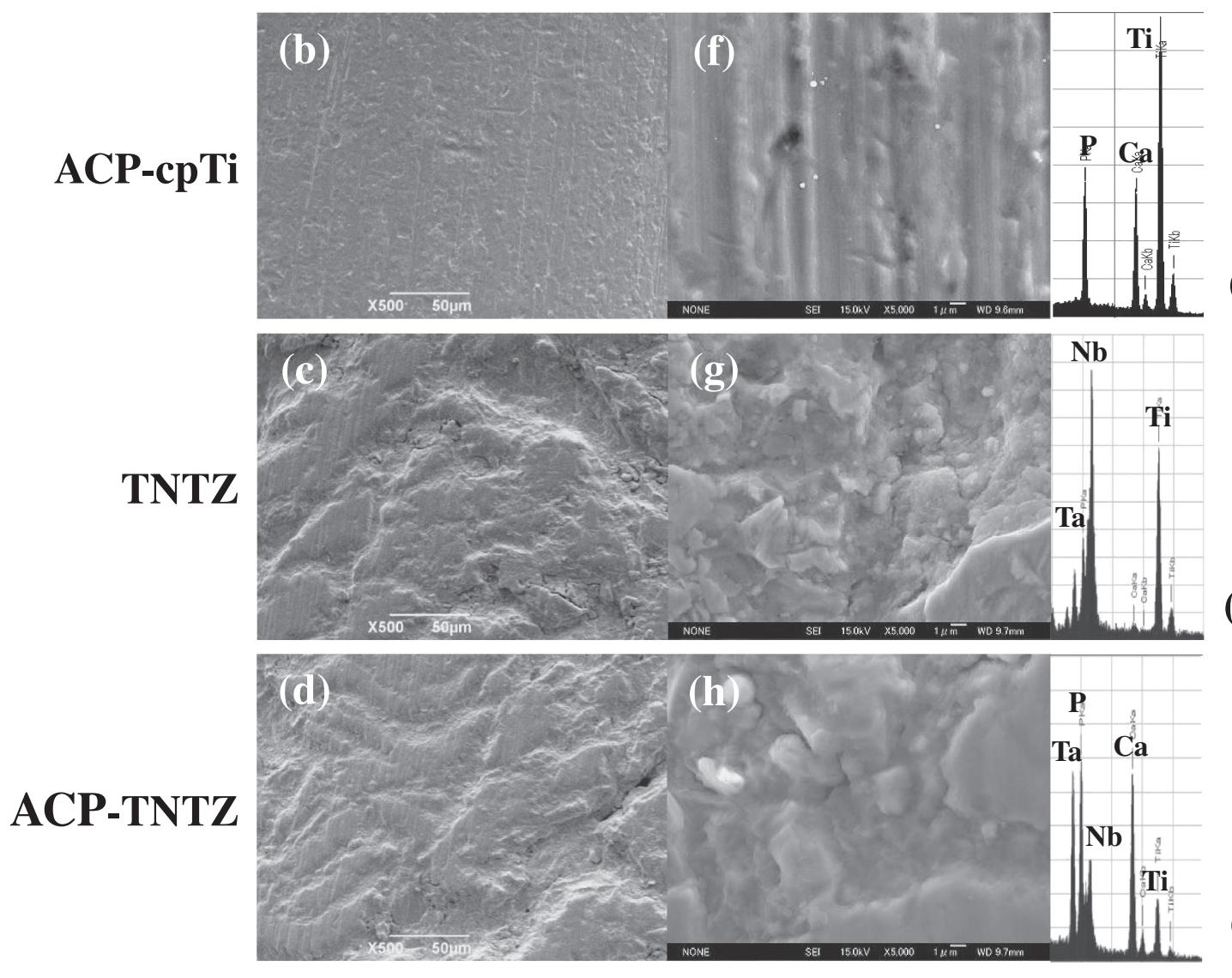

(I)

Fig. 1 SEM micrographs and EDX spectra of implant surfaces before insertion: (a), (e), (i) cpTi; (b), (f), (j) ACP-cpTi; (c), (g), (k) TNTZ; and (d), (h), (l) ACP-TNTZ. EDX spectra (i), (j), (k), (l) indicate EDX spot elemental analyses sites shown in (e), (f), (g) and (h), respectively. Bars $=50 \mu \mathrm{m}$ (a), (b), (c), (d), $1 \mu \mathrm{m}$ (e), (f), (g), (h).

\subsection{Animal experiments}

Ten-week-old male Sprague-Dawley rats were used to evaluate the osseointegration and biomechanical aspects. The experimental rats were anesthetized with an intraperitoneal injection of sodium pentobarbital $(50 \mathrm{mg} / \mathrm{kg})$ supplemented with ether inhalation. A full-thickness incision was made aseptically from the head to the medial aspect of the right femur, and exposed the distal surface of the femur. An implant cavity was prepared using a surgical drill with irrigation. Two implants per femur were placed 7 and $11 \mathrm{~mm}$ from the knee joint. The surgical site was closed in layers; muscle layers were sutured using dissolvable stitches, and skin layers were closed using non-absorbable sutures.

\subsection{Push-in tests}

To evaluate the degree of osseointegration, push-in tests were performed. ${ }^{18)}$ After the implant placements, rats were sacrificed at weeks 2, 4 and 8 of healing. The femurs were harvested and embedded in an auto-polymerizing resin, and then push-in tests were carried out on a universal testing machine (EZ-L-500N, Shimadzu Co., Kyoto, Japan).

\subsection{Analyses of the implant-tissue interface}

After the push-in tests, the exposed implants were soaked in stirred distilled water for an hour and dried at $37^{\circ} \mathrm{C}$ in a vacuum. The implant surfaces after the push-in tests were examined using scanning electron microscopy (SEM) and analyzed using energy-dispersive X-ray (EDX) spectroscopy (JSM-6500F, JEOL, Tokyo, Japan).

\subsection{Statistical analysis}

At each healing period, the push-in test values for ACPcoated and non-coated implants were compared using MannWhitney's U test. 


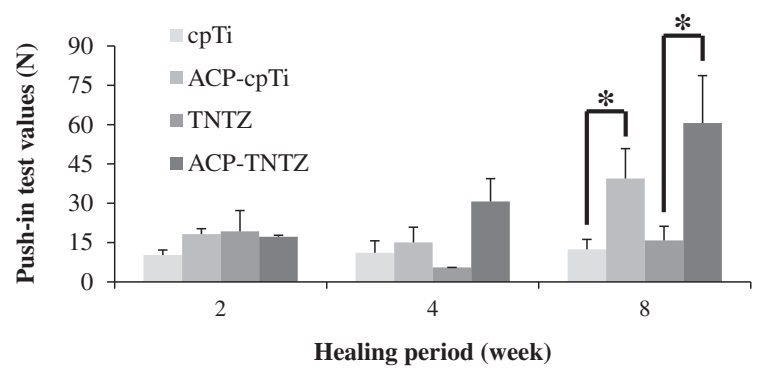

Fig. 2 Push-in test values for cpTi, ACP-cpTi, TNTZ and ACP-TNTZ implants. Data are the mean $\pm \mathrm{SD}(n=4)$. * Significant differences $(P<0.05)$ determined by the Mann-Whitney $\mathrm{U}$ test.

\section{Results}

\subsection{Surface analyses of cylindrical implants before insertion}

The implant surfaces before insertion were examined by SEM and EDX analyses (Fig. 1). In the SEM observation, there is no characteristic change by RF magnetron sputtering. In the EDX analyses, characteristic metal substrate element peaks were observed in both non-coated implants. After ACP coating, the EDX spectra of ACP-cpTi and ACP-TNTZ showed $\mathrm{Ca}$ and $\mathrm{P}$ peaks from the ACP coating, without detectable contamination, and metallic

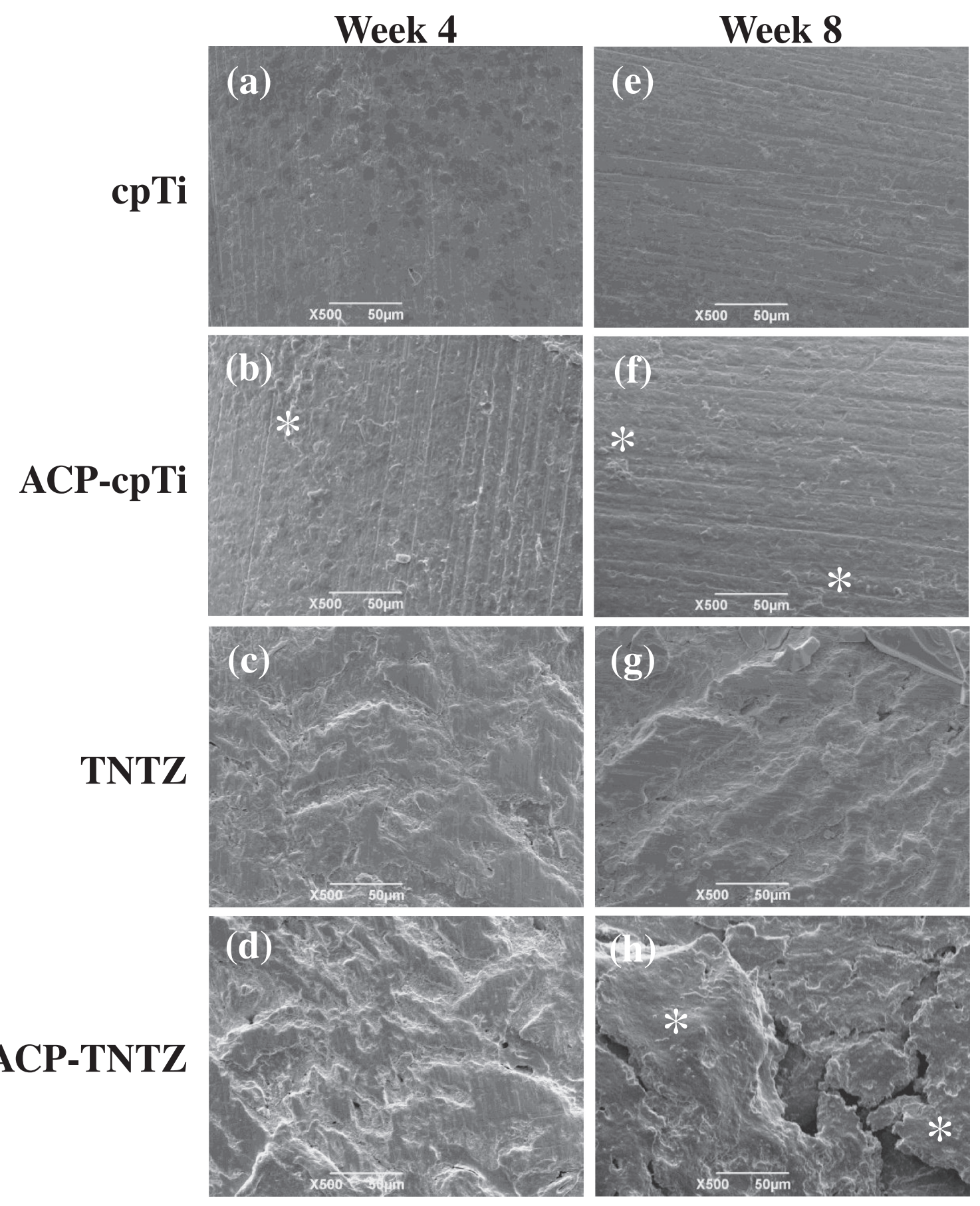

Fig. 3 SEM micrographs of cpTi, ACP-cpTi, TNTZ and ACP-TNTZ implants after push-in tests: (a), (e) cpTi; (b), (f) ACP-cpTi; (c), (g) TNTZ; and (d), (h) ACP-TNTZ. (a), (b), (c) and (d) were at healing period week 4; (e), (f), (g) and (h) were at week 8. *: remnant tissue. Bars $=50 \mu \mathrm{m}$. 


\section{ACP-cpTi}
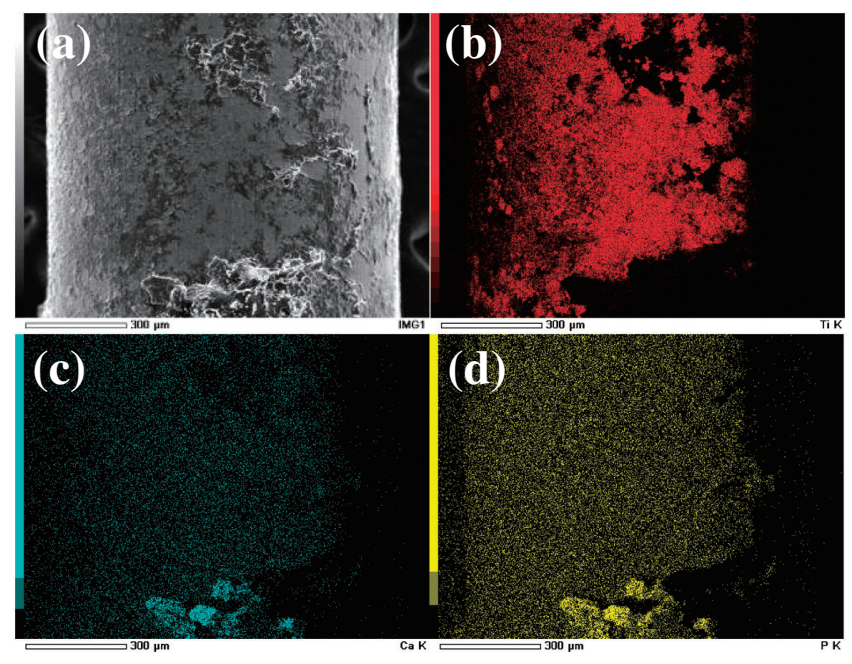

ACP-TNTZ
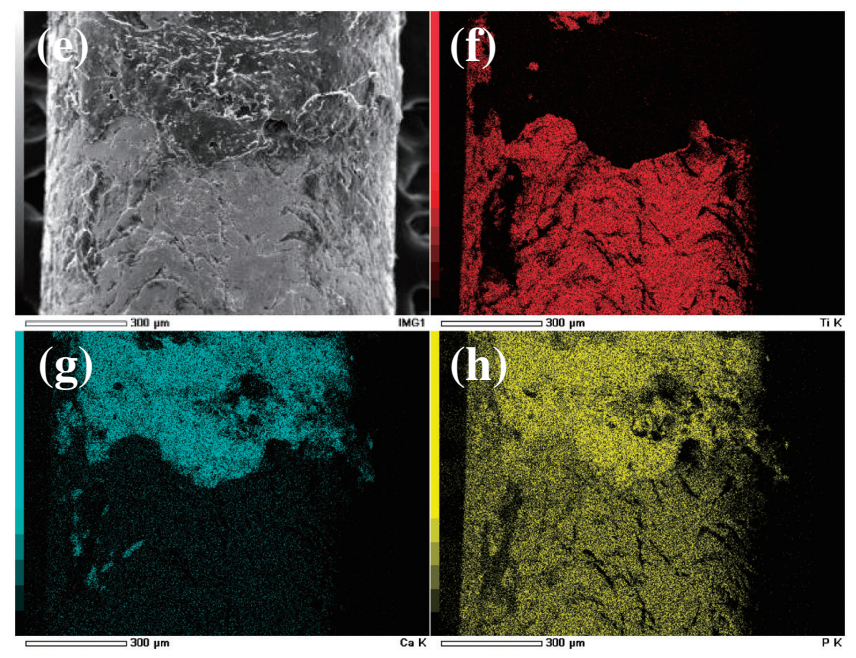

Fig. 4 EDX mapping of implant surfaces of ACP-cpTi and ACP-TNTZ at 8 weeks. (a), (b), (c), (d) ACP-cpTi implant surfaces; (e), (f), (g), (h) ACP-TNTZ implant surfaces. (a), (e) SEM micrographs; (b), (f) Ti element; (c), (g) Ca element; and (d), (h) P element. Bars $=300 \mu \mathrm{m}$.

substrate element peaks were also detected (Figs. 1(j) and $1(1))$.

\subsection{Push-in tests on animals}

At 2 and 4 weeks, there is no significant difference between the push-in test values for the ACP-coated groups and the non-coated groups. However, at 8 weeks, the values for the ACP-TNTZ implants were significantly higher than those for the non-coated TNTZ implants (Fig. 2). (Data are mean $\pm \mathrm{SD}, n=4$, Mann-Whitney's U test, $P<0.05$.)

\subsection{Analyses of the implant-tissue interface}

After the push-in tests, SEM observations (healing period weeks 4 and 8), spot elemental analyses, and mapping analyses (healing period week 8) were performed to observe the biological structures.

The surfaces of the non-coated implants were relatively smooth, without remnant tissue, similar to those before insertion (Figs. 3(a), 3(c), 3(e) and 3(g)). In contrast, the ACP-coated implant surfaces were partially covered by remnant tissue (Figs. 3(b), 3(d), 3(f) and 3(h), indicated by $\left.{ }^{*}\right)$. In particular, the amount of remnant tissue on the ACPTNTZ surfaces increased as the healing period increased (Figs. 3(d) and 3(h)).

EDX analyses of the implant surfaces of ACP-cpTi and ACP-TNTZ at 8 weeks were performed, which had the highest push-in test value; the original bone tissue was also analyzed (Figs. 4 and 5). The EDX mapping analyses showed no Ti peak (Figs. 4(c) and 4(g)). Conversely, a Ti peak was detected at the sites without Ca peaks (Figs. 4(b) and $4(\mathrm{f}))$. $P$ peaks were detected over the entire surface (Figs. 4(d) and 4(h)).

EDX spot elemental analyses revealed that $\mathrm{Ca}$ and $\mathrm{P}$ peaks were present at the sites with remnant tissue (Figs. 5(j) and 5(1)). Conversely, metallic substrate element peaks were detected at the sites without any remnant tissue (Figs. 5(i) and $5(\mathrm{k})$ ). The ratio of the Ca to $\mathrm{P}$ peaks in the remnant tissue was similar to that of the original bone element peaks (Fig. 5(h)).

\section{Discussion}

The aim of the present study was to confirm the osseointegration ability of TNTZ implants and the influence of ACP coatings prepared by RF magnetron sputtering on TNTZ implants. Our study group investigated TNTZ, developed by Niinomi, ${ }^{1)}$ as a dental implant material. First, we performed push-in tests to determine whether TNTZ has sufficient osseointegration ability. ${ }^{18)}$ Push-in tests are used to evaluate the mechanical properties of the bone-implant interface. ${ }^{19)}$ The results of the push-in tests revealed that TNTZ may be useful as a dental implant material. Furthermore, surface modification by RF magnetron sputtering could increase the degree of osseointegration in both cpTi and TNTZ implants.

It has been reported that the various synthesis routes of ACP make different formation, composition, structure and physico-chemical properties, in spite of having the similar $\mathrm{X}$-ray diffraction pattern, and their $\mathrm{Ca} / \mathrm{P}$ molar ratios are relatively wide range ( 1 to 2 or even higher). ${ }^{20)} \mathrm{ACP}$ having $\mathrm{Ca} / \mathrm{P}$ molar ratio 1.5 is recently called amorphous tricalcium phosphate (ATCP) because this ACP is considered to be consisted from TCP cluster. A previous study using ACP with $\mathrm{Ca} / \mathrm{P}$ molar ratio 1.5 synthesized chemically showed that while the ACP could be highly soluble in nature, it remains un-dissolved in vivo and is converted into apatite with rasing highly osteoconductive property. ${ }^{21)}$ However, in the case of synthesis by high energy processing techniques, the synthesized ACP also showed wide range of $\mathrm{Ca} / \mathrm{P}$ ratios but a biodegradable property in vivo, ${ }^{20)}$ suggesting that the calcium phosphates called ACP display distinct physicochemical and tissue response properties depending on the material characteristics possibly determined by the synthesis processes. EDX analyses of the implant surfaces revealed that $\mathrm{Ca} / \mathrm{P}$ atomic ratio of ACP on cpTi, TNTZ was 1.33 and 1.55 , respectively (Figs. 1(j), 1(1)). After push-in test, $\mathrm{Ca} / \mathrm{P}$ atomic ratio at the metallic surface was $0,0.26,2.20$ and 1.51 (Figs. 5(i), 5(j), 5(k) and 5(1), respectively). In addition to 

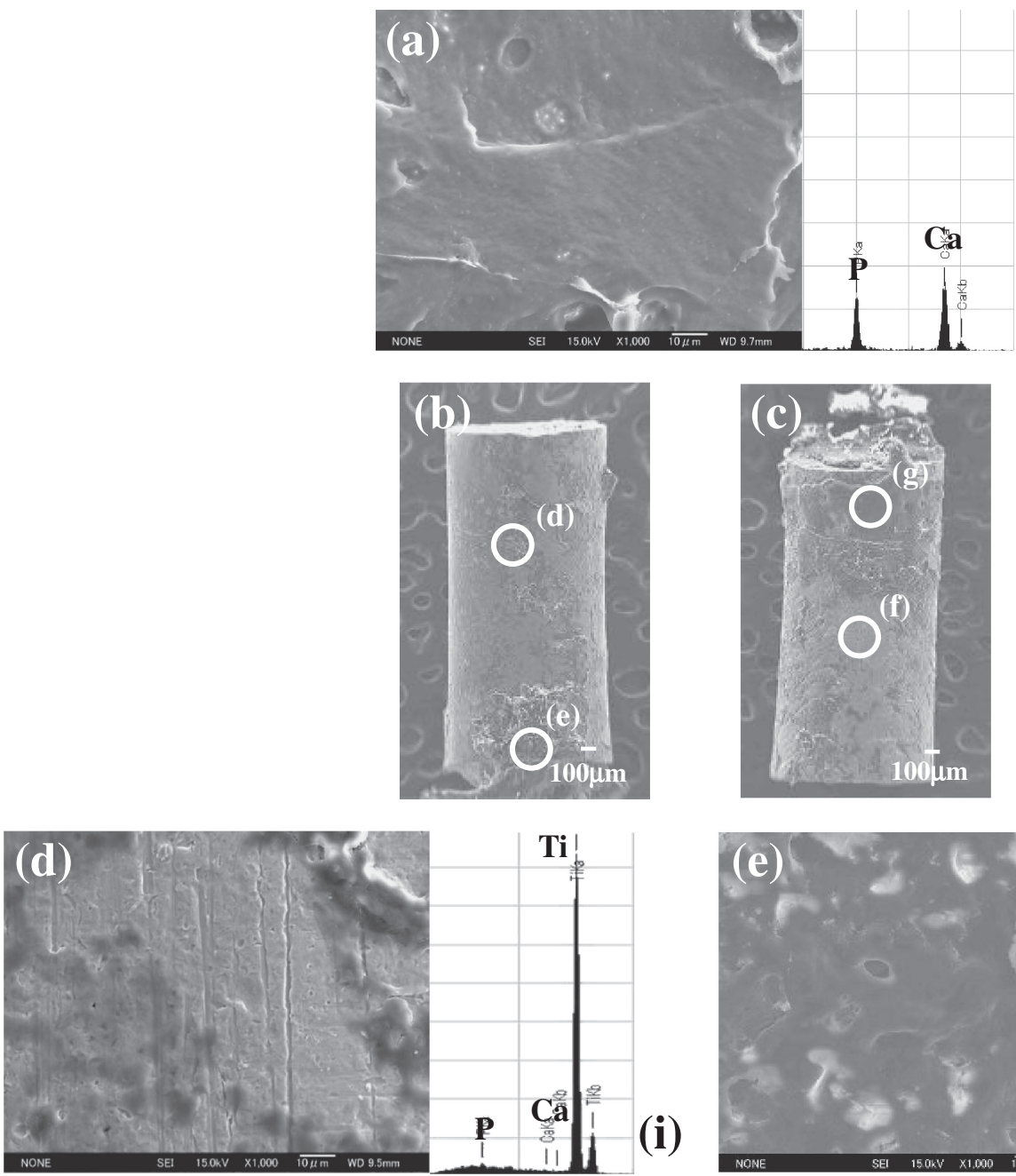

(i)
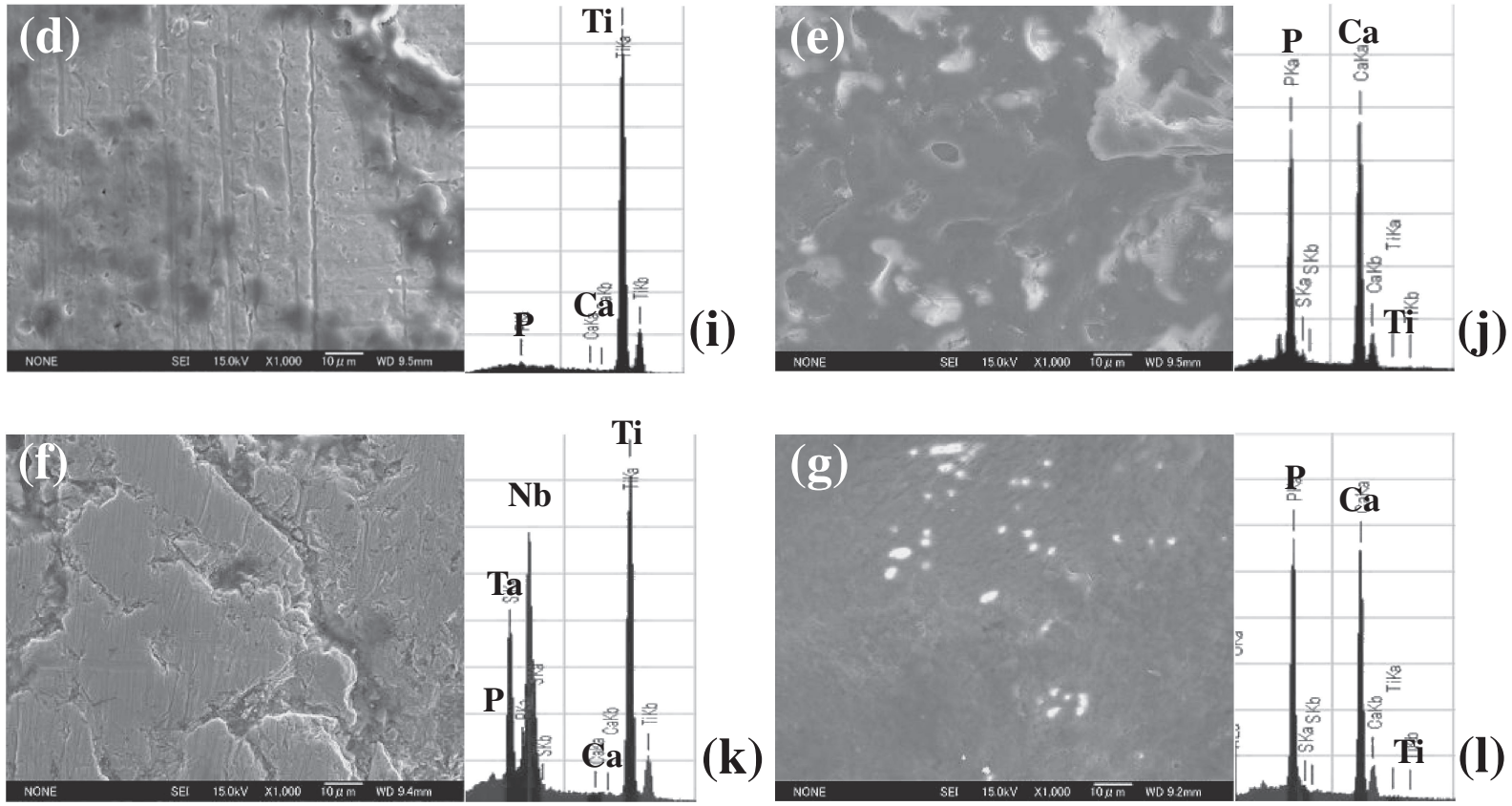

(l)

Fig. 5 SEM micrographs and EDX spectra of implant surfaces of ACP-cpTi and ACP-TNTZ at 8 weeks, and of original bone tissue. (a) Original bone tissue of 18-week-old Sprague-Dawley rats; (b), (d), (e) ACP-cpTi; and (c), (f), (g) ACP-TNTZ. (d), (e), (f) and (g) show the sites corresponding to the circles (d), (e), (f) and (g) in (b) and (c). (h), (i), (j), (k) and (l) are the EDX analyses for (a), (d), (e), (f) and (g), respectively. Bars $=10 \mu \mathrm{m}(\mathrm{a}),(\mathrm{d}),(\mathrm{e}),(\mathrm{f}),(\mathrm{g}), 100 \mu \mathrm{m}(\mathrm{b}),(\mathrm{c})$.

that, bone of 18-week-old male Sprague-Dawley was 2.0 (Fig. 5(h)). These results suggested that there were two sheared-surface patterns; one was between the metallic substrate and bone tissue, and the other was inside the bone tissue. It has been reported that resorption lacunae by osteoclasts were not observed in the case of ACP coating in vivo. ${ }^{22)}$ However, it has also been reported that ACP coatings in the tibiae of rabbits were completely resorbed and replaced by bone, which came into direct contact with underlying materials without any intervention of soft tissue until 16 weeks. ${ }^{23)}$ Ueda et al. reported that ACP coatings produced by RF magnetron sputtering rapidly dissolved in vitro. ${ }^{24)}$ Our EDX analyses data are consistent with these studies.

\section{Conclusion}

The results of this study confirm the following:

(1) TNTZ implants have the potential to achieve osseointegration to the same degree as that achieved by cpTi implants.

(2) The osseointegrations of cpTi and TNTZ implants are 
improved by surface modification with ACP coatings prepared using an RF magnetron sputtering system, especially in the later stages of healing.

\section{Acknowledgments}

This study was supported in part by Research and Education Funding for Inter-University Research Project (Highly-functional Interface Science: Innovation of Biomaterials with Highly-Functional Interface to Host and Parasite), Ministry of Education, Culture, Sports, Science and Technology (MEXT), Japan, and in part by a Grant-in-Aid for Young Scientist B (22791861 and 23792210) from MEXT, Japan.

\section{REFERENCES}

1) D. Kuroda, M. Niinomi, M. Morinaga, Y. Kato and T. Yashiro: Mater. Sci. Eng. A 243 (1998) 244-249.

2) M. Niinomi: Biomaterials 24 (2003) 2673-2683.

3) M. Niinomi, T. Hattori, K. Morikawa, T. Kasuga, A. Suzuki, H. Fukui and S. Niwa: Mater. Trans. 43 (2002) 2970-2977.

4) M. Niinomi: Bull. Iron Steel Inst. Jpn. 15 (2010) 661-670.

5) A. Meunier, P. Christel, L. Sedel, J. Witvoet and D. Blanquaert: Int. Orthop. 14 (1990) 67-73.

6) M. Niinomi: J. Mech. Behav. Biomed. Mater. I 3 (2008) 0-42.

7) M. Niinomi and M. Nakai: Int. J. Biomater. (2011) doi:10.1155/2011/ 836587.

8) P. I. Brånemark, B. O. Hansson, R. Adell, U. Breine, J. Lindström,
O. Hallén and A. Ohman: Scand. J. Plast. Reconstr. Surg. Suppl. 16 (1977) 1-132.

9) B. Rangert, P. H. Krogh, B. Langer and N. Roekel: Int. J. Oral Maxillofac. Implants 10 (1995) 326-334.

10) F. Isidor: Int. J. Oral Maxillofac. Implants 13 (1998) 377-383.

11) F. Isidor: Clin. Oral Implants Res. 8 (1997) 1-9.

12) T. Miyata, Y. Kobayashi, H. Araki, T. Ohto and K. Shin: Int. J. Oral Maxillofac. Implants 15 (2000) 425-431.

13) A. Piattelli, G. Vrespa, G. Petrone, G. Iezzi, S. Annibali and A. Scarano: J. Periodontol. 74 (2003) 385-390.

14) Y. Yang, K. H. Kimc and J. L. Ong: Biomaterials 26 (2005) 327-337.

15) T. Narushima, K. Ueda, T. Goto, H. Masumoto, T. Katsube, H. Kawamura, C. Ouchi and Y. Iguchi: Mater. Trans. 46 (2005) 22462252.

16) K. Ueda, T. Narushima, T. Goto, T. Katsube, H. Nakagawa, H. Kawamura and M. Taira: Mater. Trans. 48 (2007) 307-312.

17) K. Ueda, T. Narushima, T. Goto, T. Katsube, H. Nakagawa, H. Kawamura and M. Taira: Mater. Sci. Forum 631-632 (2010) 211-216.

18) T. Ogawa, S. Ozawa, J.-H. Shih, K. H. Ryu, C. Sukotjo, J.-M. Yang and I. Nishimura: J. Dent. Res. 79 (2000) 1857-1863.

19) W. J. Seong, S. Grami, S. C. Jeong, H. J. Conrad and J. S. Hodges: Clin. Implant Dent. Relat. Res. 26 (2011) 826-836.

20) C. Combes and C. Rey: Acta Biomater. 6 (2010) 3362-3378.

21) O. Suzuki, M. Nakamura, Y. Miyasaka, M. Kagayama and M. Sakurai: Tohoku J. Exp. Med. 164 (1991) 37-50.

22) S. Leeuwenburgh, P. Layrolle, F. Barrere, J. de Bruijn, J. Schoonman, C. A. van Blitterswijk and K. de Groot: J. Biomed. Mater. Res. 56 (2001) 208-215.

23) M. Nagano, T. Nakamura and T. Kokubo: Biomaterials 17 (1996) 1771-1777.

24) K. Ueda, T. Narushima, T. Goto, M. Taira and T. Katsube: Biomed. Mater. 2 (2007) S160-S166. 